\title{
Chemical Leaching of Low Rank Coal and its Characterization using SEM/EDAX and FTIR
}

\author{
B. Manoj*, A.G. Kunjomana and K.A. Chandrasekharan \\ Department of Physics, Christ University, Hosur Road, Bangalore -29 \\ Karnataka, India. \\ *Corresponding Author: manoj.b@christuniversity.in
}

\begin{abstract}
Demineralization of coal carried out using EDTA for different time and HF of varying concentration. The residual coal from each treatment was characterized together with virgin coal using Scanning Electron Microscopy and energy dispersive X-ray analysis (EDAX). Micrograph and EDAX analysis of Virgin coal reveal the features lithophiles like aluminium, silicates and calcium. The absence of the features corresponding inorganic elements in residual samples is an indication of demineralization. An elemental analyzer was adopted to analyze CHNS on virgin and residual coal sample. The current research compares the leaching efficiency of a mild leachant and a strong leachant. The final analysis showed that the coal under study was sub bituminous coal and leaching could improve the amount of carbonaceous material.
\end{abstract}

Keywords: Coal; Minerals; Characterization; SEM; EDAX; FTIR

\section{INTRODUCTION}

Study on various non renewable energy sources and enhancement of their fuel efficiency through different processes like chemical and biological means is a topic of continuing research. Many environmentalists see coal as inherently dirty. Coal minerals are considered objectionable due to the process as well as environmental problems. Coal minerals might be epigenetic and syngentic. Both constitute the inorganic part of the coal and if its concentration increases above a certain level, it is hazardous to the environment and the furnace. Minerals are needed to certain levels due to the catalytic effects in gasification and liquefaction [1-2]. Efforts are needed to reduce the ash forming inorganic elements and develop clean methods of using coal. Demineralization prior to utilization is an effective way to ensure environmental friendly combustion of coal as a fuel. 
Many techniques are employed for coal beneficiation in order to demineralize and desulphurise coal [1-7]. Among these methods, leaching of coal with different leachants is presently carried out worldwide [1, 6-7]. Mineral acids are generally used to demineralize the coals but due to their strong oxidizing power, they modify the coal surface morphology, harm the carbon, reduce the calorific value and create environmental problems. Mild leachants are generally used to avoid such disadvantages.

In the present investigation, coal sample was collected from Godavari coal field, characterized and then subjected to leaching with leachants like HF and EDTA. The objective was to reduce mineral content and sulphur to overcome deleterious effects associated with combustion of low rank coal, without harming the carbon content of coal and compare the leachability of EDTA with mineral acid HF.

\section{MATERIALS AND METHODS}

\subsection{Preparation of Coal Sample}

The sample was randomly hand picked from a mine in Godavari coal field. It was crushed and ground in a pestle and mortar, screened through 250 micrometer sieves using a sieve shaker. The definite sized coal sample was dried in a vaccum oven at $70^{\circ} \mathrm{C}$ for one hour and cooled in a dessicator. The ultimate analysis of the virgin coal and residual coal under study is provided in Table 1 .

\subsection{Extraction Procedure}

Five grams portion of the coal sample under study was extracted separately with EDTA and HF. The specified amount of coal was slurried in $50 \mathrm{ml}$ of extracting solution in a beaker and stirred for time duration of 60 minutes at $30^{\circ} \mathrm{C}$. The beaker was maintained at room temperature. After being treated for the specified time, the slurry was allowed to settle down and the precipitate was removed. The slurry was filtered using filter paper to remove the capture solution. The residual coal was washed in a water column of distilled water for one day and filtered again. The filtrates was dried at temperature of $80^{\circ} \mathrm{C}$ and allowed to cool slowly in a dessicator. The sample was treated with $0.1 \mathrm{~N}$ EDTA for a period of $24 \mathrm{hrs}, 48 \mathrm{hrs}$, $72 \mathrm{hrs}$ and $96 \mathrm{hrs}$. The residue was washed with distilled water and dried again at about $80^{\circ} \mathrm{C}$. The powdered sample was treated with Hydrofluoric acid (HF) of varying concentration $(40 \%, 30 \%, 20 \%$ and $10 \%)$ for $24 \mathrm{hrs}$ and the slurry was filtered and dried as discussed above.

The use of Scanning electron microscopy (SEM), Energy dispersive X-ray (EDAX) analysis and FTIR has many current and potential applications for the coal and mineral processing industries. It is used to investigate the formation and deposition of unwanted ash by- products and in analyzing substances of environmental concern. 


\subsection{EDAX Analysis}

Energy dispersive X-ray spectrophotometer (EDAX) model JSM 840A from JEOL Company in Japan was used for the mineral analysis of treated and virgin coals.

\subsection{SEM Analysis}

The SEM micrographs of the virgin and residual coal samples obtained by Scanning Electron Microscope (SEM) model JSM 6390 from JEOL Company in Japan.

\subsection{FTIR Analysis}

For analysis, about $20 \mathrm{~g}$ of the sample was crushed in to fine powder of about $5 \mu \mathrm{m}$ in size. $2 \mathrm{mg}$ of the powdered sample was then mixed with $\mathrm{KBr}$ in the ratio 1:200 and ground for about 10 minutes. The grinding time and the sample to $\mathrm{KBr}$ ratio 1:200 were kept constant for all the samples under study to ensure uniformity. The mixture was then placed in a $13 \mathrm{~mm}$ diameter die cavity and pressed under high pressure. The spectrum was recorded by using Shimadzu FTIR -8400 spectrometer in the region $4000-400 \mathrm{~cm}^{-1}$. This spectrometer had the resolution of $4 \mathrm{~cm}^{-1}$. To obtain consistent records the FT-IR spectra was recorded in 20 scan mode. The FTIR spectrum of virgin coal and residual coal after chemical leaching was shown in Figure 7 and Figure 8.

\subsection{Elemental Analysis}

Elemental analysis was carried out using VarioEL III CHNS analyzer. The analysis was able to determine CHNS on dried samples. Oxygen content was obtained subtracting the total weight of the Carbon, Hydrogen, Nitrogen and sulphur from the total.

\section{RESULT AND DISCUSSION}

Energy dispersive X-ray spectrophotometric (EDAX) study of the virgin coal and residue was conducted to study and compare the leachability of various leachants used. EDAX spectra of virgin sample and treated coals were given in Figure.1-3. It was evident from the Figure.1 and CHNS analysis (table.1) that the coal under study had Carbon (60.12\%), Oxygen (31.107\%), $\mathrm{Al}(25.66 \%), \mathrm{Si}(53.87 \%), \mathrm{S}(9.04 \%)$ and $\mathrm{Ca}(11.43 \%)$. It was observed from the Ultimate study that the Carbon content increased to a maximum of $69.44 \%$ when leached with EDTA for 24hrs. It was also inferred from the EDAX analysis (Figure.2) that lithophilic elements like $\mathrm{Ca}$ was completely removed where as Sulphur content was reduced considerably $[1,4,6,8]$. It was also noticed that the aluminium and silicates content did not decrease with EDTA leaching. 

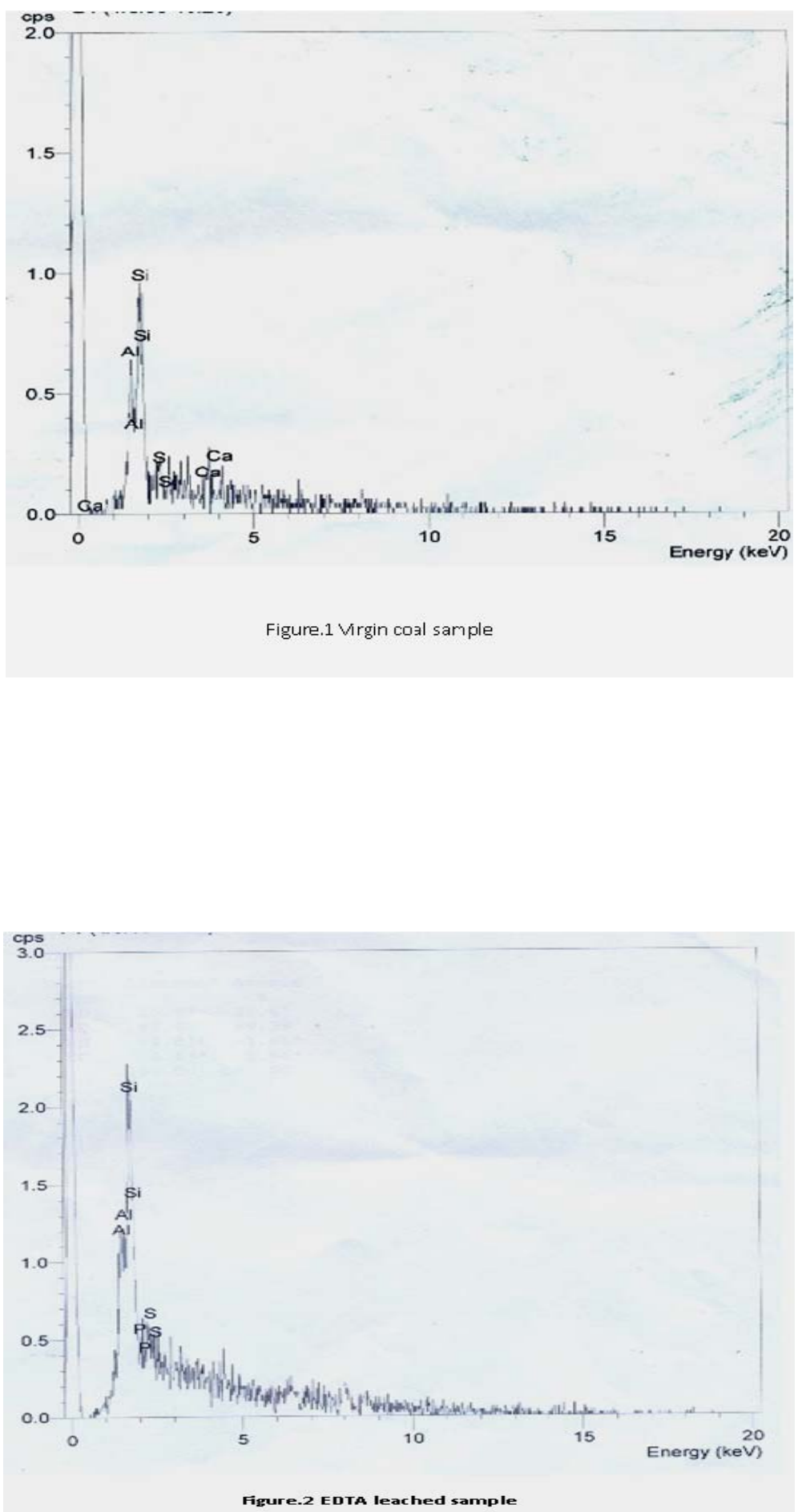


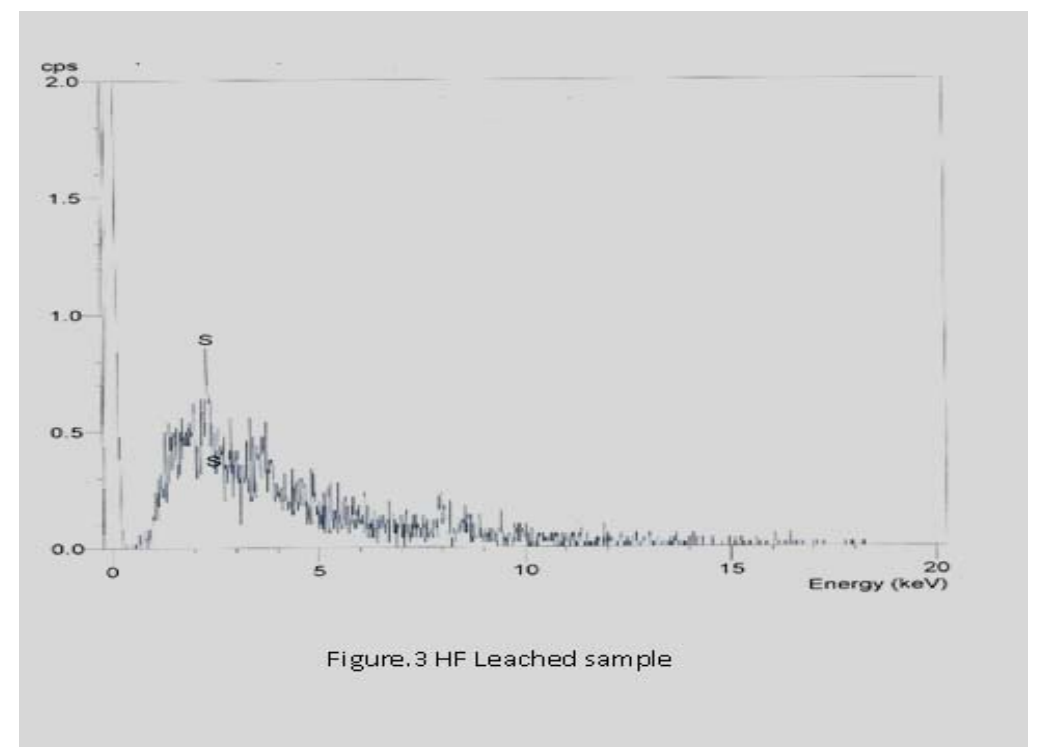

When the sample was leached with strong leachant like HF (Figure.3), almost all minerals (Silicates, Aluminates and Calcites) were removed and only a trace amount of sulphur was present in the sample. It was matched with CHNS analysis where the sulphur content was reported to be at very low level of $0.016 \%$. During the leaching the carbon content showed an increase of $28.842 \%$ from the initial value.

\subsection{SEM Observation}

The SEM micrographs of the virgin and variously leached samples were provided in Figures.4-6. Figure.4 represents the SEM image of the virgin coal sample. A bulk microstructure composed of homogeneously distributed network of small crystallites showed the presence of minerals. In the matrix, luminous as well as non luminous features could be seen. These features indicate the presence of minerals distributed in the organic matrix. Many fissures, cleats, cracks and veins were also observed. The bright luminosity was due to the presence of aluminium,potassium or sodium. The dark luminosity was mainly due to the presence of chalcophiles $[1,5]$. Etiched pits, layers, some islands and hills \& valleys could also be seen randomly distributed throught the micrograph. These might had resulted from the calcinations of dolomite and calcites or their assemblages due to thermal shock during metamorphism. It was inferred that the coal under study contain large proportins of Silica, Calcium carbonates and Dolomite, as well as some proportions of elements such as aluminium, potassium and sulphur.

To remove the minerals and enrich the coal in usable carbon, chemical leaching was performed. The sample was leached with a chelating agent (EDTA) for 24 hours and the SEM of the residual coal was taken. 


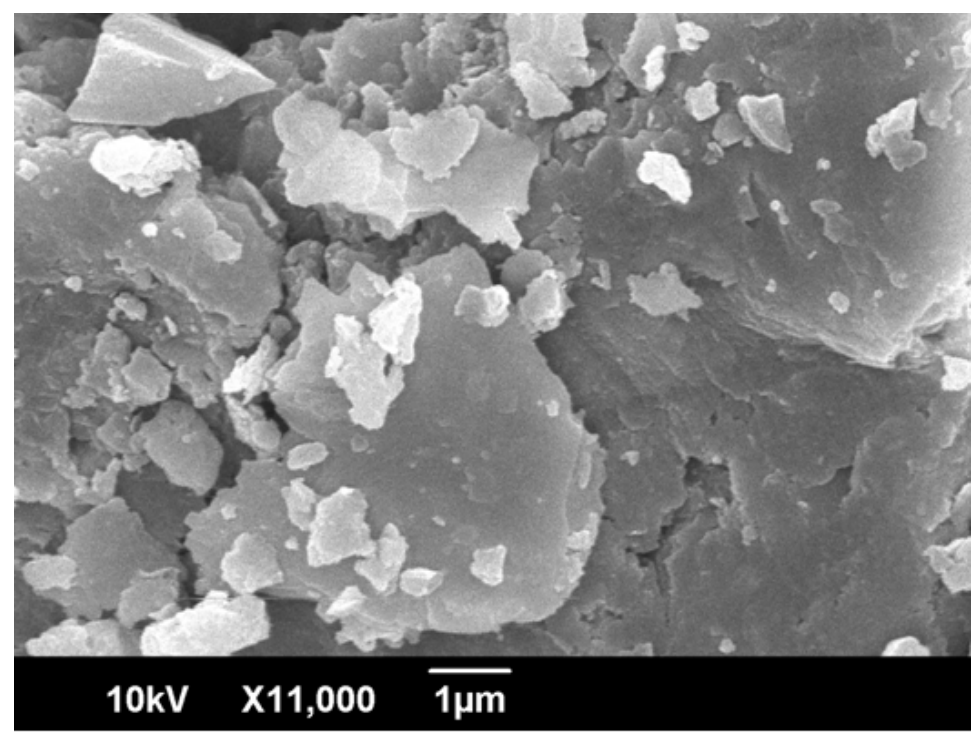

Figure.4 SEM micrograph of virgin coal sample

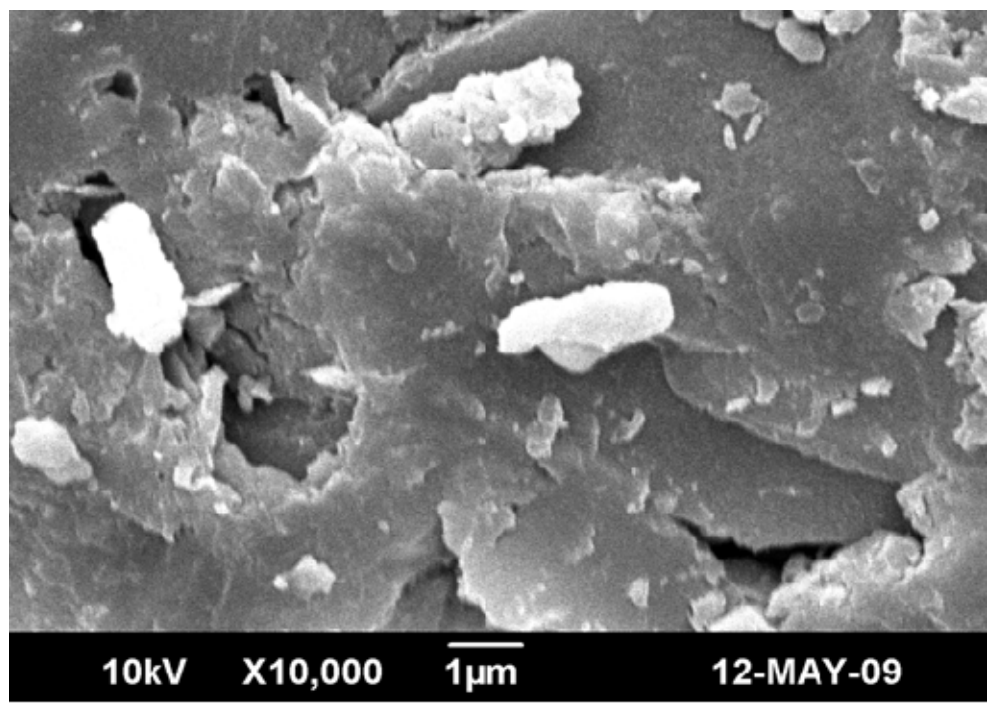

FIgure. 5 SEM mIcrograpt of EDTA. leached sample

The SEM study (Figure 5) revealed that the EDTA leaching did not harm the Carbon. From the EDAX study it was also noticed that the mineral content especially the sulphur content decreases to minimum when treated with EDTA. Numerous aggregated particle could be seen on the suface. The increase of porosity was an indication that significant amount of inorganic elements were being removed. However, the surface coverage was still bright and luminous indicating the presence of mineral phases. 
Leaching was also performed with Hydrofluric acid (HF). The SEM image of the residual coal from this treatment was provided in Figure 6. It could be seen that this leahant caused morpholigical changes in the particle and did enormous harm to the surface by leaching many of the inorganic elements.

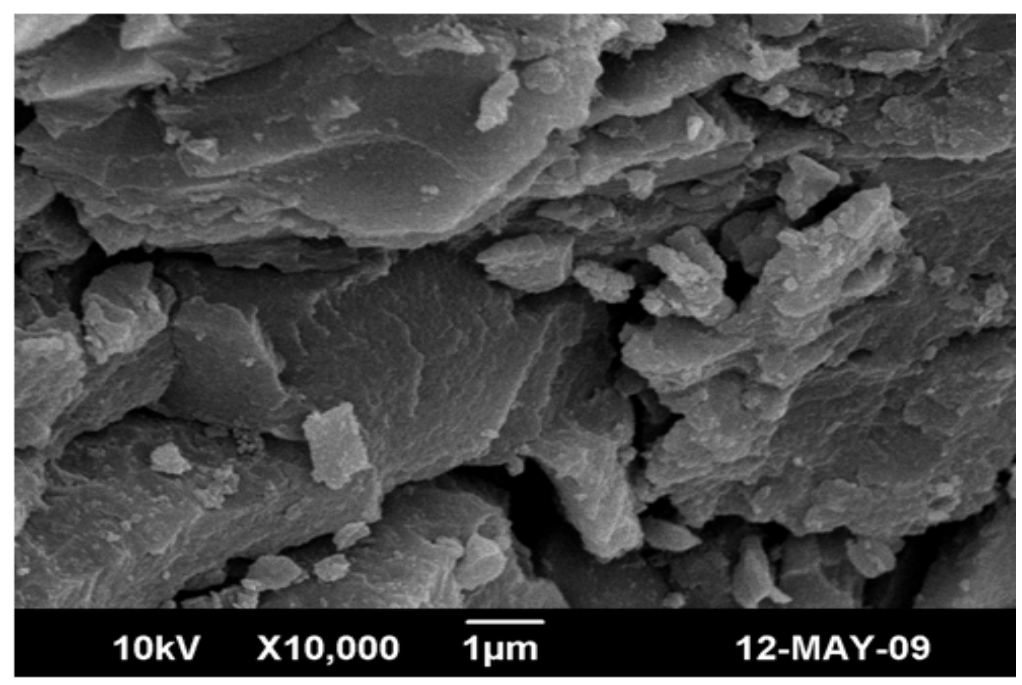

Figure.6 SEM micrograph of HF leached sample

Upon comparing different micrograph (Figure 4-Figure 6), it can be concluded that amongst the leachants used, HF was very effective for removing mineral matter like silicates and aluminates. EDTA leaching could remove calcites completely and leach the sulphur content partially.

Table 1 showed that demineralization using HF and EDTA leaching modified the Ultimate and EDAX analyis of the sample. There was an appreciable reduction in the Sulphur and Oxygen content from the virgin sample [6-7]. EDTA could remove certain amount of minerals and oxygenated functional groups from the sample. 
Table.1 EDAX analysis and Ultimate Analysis

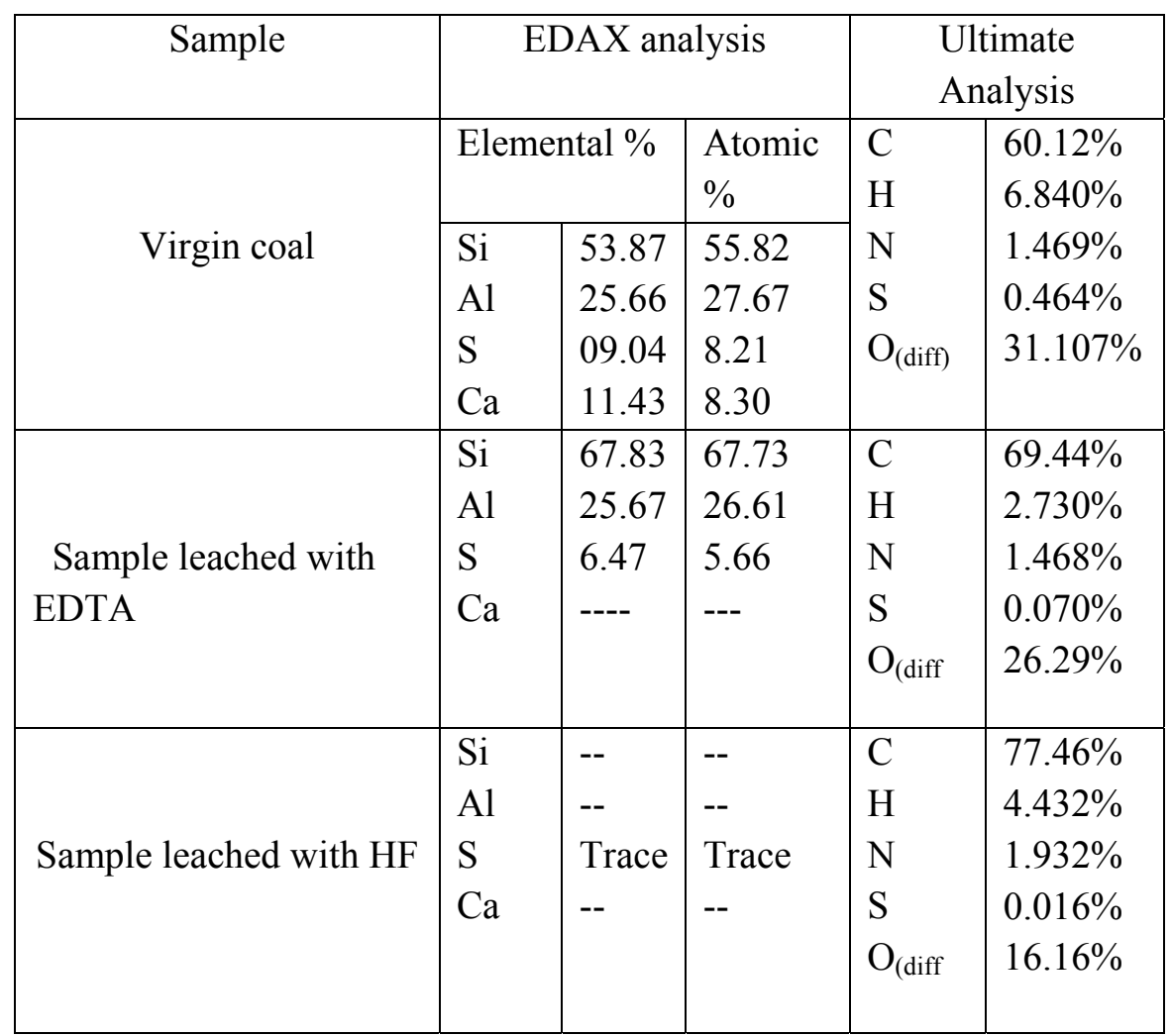

\subsection{FTIR Analysis}

Each absorption band in the spectra (Figure 7) was analyzed for the intensity of transmission by Lambert-Beer law [9-12]. Bands were assigned according to the published articles [9-12]. Figure.7 showed that the entire spectrum had more or less similar broad characteristic absorption bands. All the absorption bands were unresolved indicating that the material constituents had either large particle size or contained polymeric units. From Figure. 8 it was observed that absorption peaks were better resolved and it was an indication of the effect of chemical treatment on the structure of coal. 


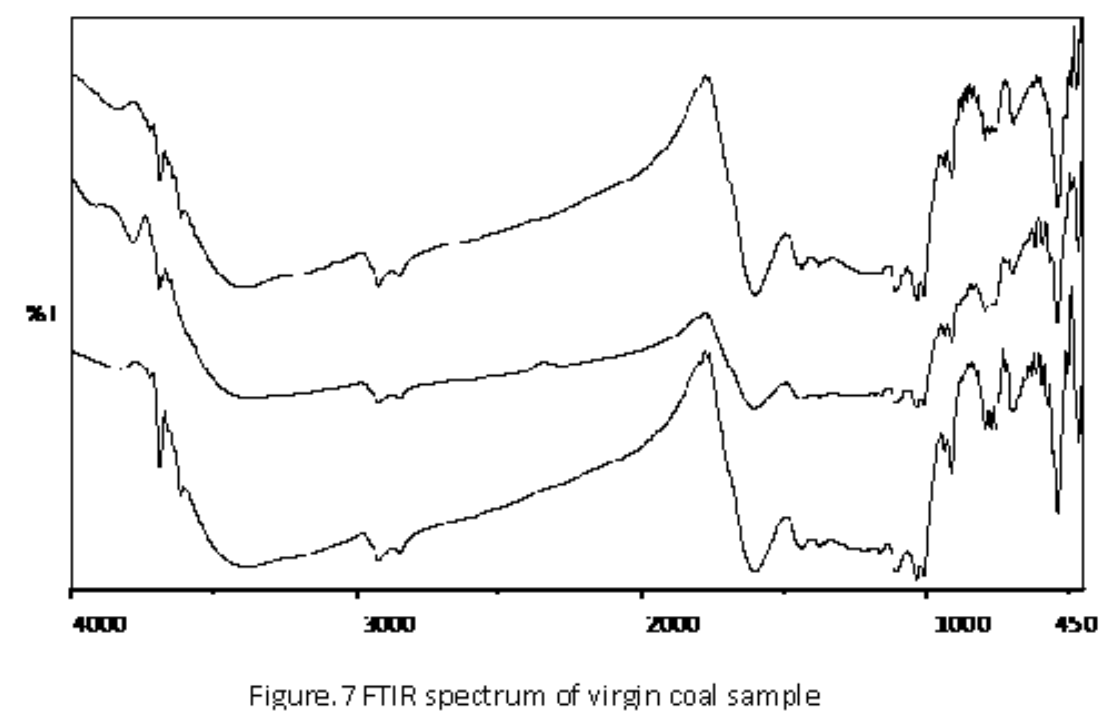

FT-IR spectrum analysis was used to investigate variations in the functional groups of coal sample used in the experiments. FTIR technique is a useful analytical method for the identification of constituents of demineralized coal samples. The spectra of the residual coal samples could be analysed in terms of the fixed mix of functional groups. $\mathrm{OH}$ and $\mathrm{NH}$ stretching in between $3100-3500 \mathrm{~cm}^{-1}$, C-H aliphatic in between $2800-3000 \mathrm{~cm}^{-1} . \mathrm{C}=\mathrm{O}$ and C$\mathrm{O}$ stretching in between $1640-1750 \mathrm{~cm}^{-1}$ attributed to phenolic ester, carboxylic acid and conjugated ketonic structures. The region from $1000-1100 \mathrm{~cm}^{-1}$ is attributed for silicate minerals. The region in between $700-900 \mathrm{~cm}^{-1}$ contains various bands related to aromatic, out of plane $\mathrm{C}-\mathrm{H}$ bending with different degrees of substitution. The change in absorption and frequency in the spectrum peaks shows how the leaching conditions affect the structure of coal.

In Figure 8 spectrums have general broad characteristics. The bands at $2800-2920 \mathrm{~cm}^{-1}(\mathrm{C}-\mathrm{H}$ aliphatic stretching) and $1375-1465 \mathrm{~cm}^{-1}(\mathrm{C}-\mathrm{H}$ aliphatic bending) are more intense in $\mathrm{HF}$ treated samples. There was absence of peaks in the carboxylic region $1640-1750 \mathrm{~cm}^{-1}$, which were attributed to phenolic structure. When concentration of HF was increased from $10 \%$ to $30 \%$ (HF10-HF30) the frequency of the $\mathrm{OH}$ band changed from $3394 \mathrm{~cm}^{-1}$ to $3445 \mathrm{~cm}^{-1}$.Upon comparing the $\mathrm{C}=\mathrm{C}$ band at $1600 \mathrm{~cm}^{-1}$, in Figure.8, spectrum corresponds to $\mathrm{HF} 20 \%$ showed maximum intensity of absorption at $1600 \mathrm{~cm}^{-1}$. The spectrum EDTA $24 \mathrm{hrs}$ also showed maximum absorption at the same frequency. This indicated that the degree of carbonization was higher when treated with HF $20 \%$ followed by EDTA $24 \mathrm{hrs}$. The band between $1100-$ $1450 \mathrm{~cm}^{-1}$ more or less remains unaffected with leaching except with HF30\% and EDTA $72 \mathrm{hrs}$. This band reduced its intensity to a minimum on these two spectrums. Leaching with EDTA could reduce the intensity of this complex molecule and most effective when treated for 72 hrs. EDTA and mineral acid (HF) were effective in solubilizing this complex molecule. 
On comparing the silicate band at $1010 \mathrm{~cm}^{-1}, 1030 \mathrm{~cm}^{-1}$ and $1090 \mathrm{~cm}^{-1}$, maximum removal was taken place with HF $20 \%$ as leachant. EDTA could not remove the silicates completely where as HF being good corrosive acid could completely demineralize the silicates. The bands between $700-900 \mathrm{~cm}^{-1}$, was due to aromatic, out of plane $\mathrm{C}-\mathrm{H}$ bending with different degrees of freedom showed very week intensity of absorption in spectrum of EDTA solubilized product compared to HF leaching. There was decrease in the spectral intensity as the time of EDTA leaching was increased. The weak absorption band at $1350 \mathrm{~cm}^{-1}$ and $1150 \mathrm{~cm}^{-1}$ was asymmetric and symmetric stretch of sulphonic acid. With treatment of HF, the intensity of this band substantially reduced, where as with EDTA the effect was not noticeable. On comparing with the FT-IR spectra of chemical solubilized coal sample (Figure.8), it was found that the hydroxyl stretching vibrations $\left(3670-3230 \mathrm{~cm}^{-1}\right)$ and $\left(1410-1310 \mathrm{~cm}^{-1}\right)$ decreases its intensity. This may be due to the reduction in oxygen functional group while leaching with acid. The aliphatic $\mathrm{CH}$ stretching vibrations $\left(2980-2845 \mathrm{~cm}^{-1}\right)$ asymmetric $\mathrm{CH}_{2}$ and $\mathrm{CH}_{3}$ bending vibrations $\left(1485-1400 \mathrm{~cm}^{-1}\right)$ decreases its intensity moderately.

The aromatic $\mathrm{C}=\mathrm{C}$ stretching vibrations $\left(1635-1600 \mathrm{~cm}^{-1}\right)$ showed strong absorption. This indicates the product was having more carbon content. This result was confirmed with the CHNS analysis. The possible reason could be a reduction of oxygen content via transformation of $\mathrm{C}=\mathrm{O}$ to $\mathrm{CH}_{2}$ or possibly via decarboxylation of the matrix, which in turn would improve Carbon and Hydrogen content and hence calorific value. Up on comparing the spectrum, it was observed that all the samples showed a remarkable absorption near $1440 \mathrm{~cm}^{-1}$ with maximum absorption for HF20\%, then EDTA 24hrs. This indicated the strong presence of methylene and methyl groups in the sample. It was not so prominent in the Chemical leached samples with HF (30\%) and HF (40\%) sample. This could be due to the enhancement of methylene absorption by the strong acid. The bands at $1541 \mathrm{~cm}^{-1}$ and 1442 $\mathrm{cm}^{-1}$ is normally present in immature coals with more lignin content. This band was shifted from strong absorption to medium intensity in the spectra of solubilized product. This revealed the effectiveness of HF leaching. The band at $1375 \mathrm{~cm}^{-1}$ is due to absorption of methyl symmetric bending vibrations in tertiary butyl groups. This band was normally present in low rank coals. The intensity of this band decreases in the solubilized product. This indicates that the leaching has improved the quality of fuel. 


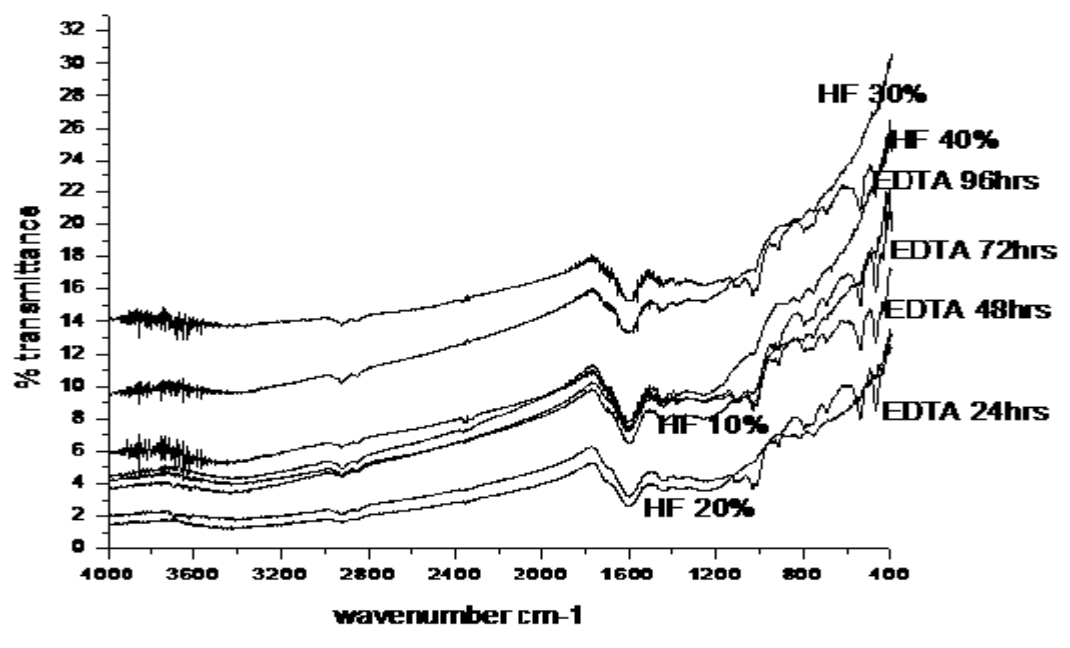

Figure.8 FTIR spectrum of HF and EDTA leached sample

\section{CONCLUSION}

It was inferred from the EDAX analysis that EDTA and HF leached the lithophilic elements like calcium effectively. The EDTA leaching could partially remove sulphur content from the sample where as the HF could remove a majority of sulphur from the sample. It was also observed that HF was a successful leachant for leaching silicates, aluminates and calcites from the sample. From the CHNS analysis it was clear that leaching could decrease the sulphur and oxygen content of the sample while increasing the carbon content.

Bauxite and silicate minerals were most abundant in the sample along with calcites and a trace of sulphur. It showed a dense, shining and blocky structure by the SEM analyses. The SEM image of the residual coal from HF treatment revealed that this leachant had caused morphplogical changes in the sample and did enormous change to the surface by leaching many of the inorganic elements. In the SEM of residual sample leached with EDTA, numerous aggregated particle could be seen. The increase of porosity was an indication that significant amount of inorganic elements were being removed. However, the surface coverage was still bright and luminous indicating the presence of mineral phases.Upon comparing different micrographs, it was concluded that amongst the leachants used HF was very effective in leaching mineral matter than EDTA. This result was in good agreement with the findings of EDAX and ultimate analysis.

On comparing the FTIR spectrum, it is inferred that the prolonged leaching with EDTA could remove more minerals from the surface. The lignin content decreased during leaching. Comparing the chemical leaching, HF was found to be a better leachant for leaching minerals from coal.

\section{ACKNOWLEDGEMENTS}


The authors are thankful to Christ University, Bangalore for the providing research facility to carry out this work. We are grateful to the Research development centre, Bharathiar University for allowing registering for $\mathrm{PhD}$ work.

\section{REFERENCES}

[1] Mohammad,Shakirullah., Imtia Ahmad., Mohammad Arsala Khan., Mohammad Ishaq., Habib ur Rehman, and Uzma Khan., 2006. "Leaching of Minerals in Degari Coal." Journal of Minerals \& Material Characterization and Engineering, Vol.5, No. 2, pp.131142.

[2] Öztas,N. A., and Yürüm, Y., 2000. "Pyrolysis of Turkish Zonguldak bituminous coal. Part 1 Effect of mineral matter." Fuel, Vol.79, pp.1221- 1227.

[3] Qing-feng, XUE., and Sheng- gao, LU., 2008. "Microstructure of ferrospheres in fly ashes: SEM, EDX and ESEM analysis”. J Zhejiang Univ Sci A, Vol.9 No. 11, pp. 1595 1600.

[4] Shakirullah, M., Ahmad, I., Ishaq, M., Rehman, H., Khan, U., and Ullah, H., 2006. "Effective Chemical Leaching and Ash Depletion of low rank coal with EDTA and Citric acid". Jour.Chem.Soc.Pak, Vol. 28, No. 1, pp. 56-61.

[5] Chin-min Cheng., 2005. "Leaching of Coal combustion products Field and Laboratory studies." PhD thesis, Ohio state University, pp. 110-181.

[6] Wenfeng Wang., Yong Qin., Shuxun Sang., Bo Jiang., Yingming Zhu and Yinghai Guo., 2007. "Sulphur variability and element geochemistry of the No.11 coal seam from the Antaibao mining district, China." Fuel, Vol.86, pp. 777-784.

[7] Rubiera, F., Arenillas, A., Arias, B., Pis, J.J., Suarez-Ruiz, I., Steel, K.M., and Patrick, J.W., 2003. "Combustion behavoiur of Ultra clean coal obtained by chemical demineralization.” Fuel, Vol.82, pp. 2145-2151.

[8] Tao Wu., Mei Gong., Ed Lester., Fuchen Wang., Zhijie Zhou and Zunbong Y., 2007. "Characterization of residual carbon from entrained-bed coal water slurry gasifier" Fuel, Vol.86, pp. 972-982.

[9] Supaluknari S., Larkins F.P, Redlich P., Jackson W.R., 1998. “An FTIR Study of Australian Coals: Characterization of Oxygen Functional Groups.” Fuel Proc. Technol. Vol.19, pp. 123-140.

[10] Rubiera, F., Arenillas, A., and Pevida, C.,2000. "Coal structure and reactivity changes induced by chemical demineralization.” Fuel processing Technology, Vol.79, pp.273279.

[11] Venkatachalapathy, R., and Ramaswamy, K.,1991. "Infrared spectroscopic study of Neyveli Lignite Samples from Mine Cut-1 and II.” Spectroscopy Letters, Vol. 24(6), pp.759 -778.

[12] Starsinic M., Otake Yoshinobu., WalkerJr P L. and, Paul C painter., 1984.”Application of FT-ir spectroscopy to the determination of COOH groups in coal." Fuel, Vol. 63(7), pp. $1002-1007$. 